\title{
Joint Beamforming and Power Control Technique for Femtocell Networks
}

\author{
Berna Özbek $^{1}$ - Uğur Bayrak ${ }^{1}$
}

Published online: 12 April 2017

(C) Springer Science+Business Media New York 2017

\begin{abstract}
The need for high data rate increases with the growing demand for wireless communication. In order to meet this demand, one of the most effective ways is deployment of small cells which are considered as a promising technique for future wireless networks. However, the deployment of these low-power base stations brings many challenges. Interference management will be one of the major drawbacks for the deployment of small cells in coverage of the macro base stations. In order to cope with interference problem, we propose a joint beamforming and power allocation technique for a two-tier network system involving femtocell and macrocell layers to design power efficient networks. The beamforming technique is applied by using partial zero-forcing method to remove the highest cross-tier interference while satisfying the macro and femtocell users' data rate requirements. Then, we perform power allocation to further reduce interference and design power efficient femtocell networks. The performance results of the proposed technique are illustrated in terms of power consumption in femtocell networks.
\end{abstract}

Keywords Interference management · Femtocell networks · Beamforming · Power control · Power efficiency

\section{Introduction}

The demand for higher data rates which increases exponentially with the usage of mobile devices will be satisfied by deploying of low power base stations and increasing the channel reuse in the wireless networks while reducing the power consumption [1]. In

Berna Özbek

bernaozbek@iyte.edu.tr

Uğur Bayrak

ugurbayrak@iyte.edu.tr

1 Department of Electrical and Electronics Engineering, İzmir Institute of Technology, Izmir, Turkey 
accordance with the recent surveys, $50 \%$ of phone calls and $70 \%$ of data services will take place indoors in the next years. Therefore, it is important for cellular networks to provide good quality coverage to indoor users. Macro base stations have drawbacks for indoor communication due to the penetration loss, which reduces the overall system throughput. In order to meet the needs of high data rate network, the deployment of a great number of macro base stations is not efficient [2].

One solution for indoor coverage, expected to be one of the most-promising technologies, is femtocells which are small and low power nodes to increase system capacity by offloading the data from macro cells to small cells [3]. These nodes, known as femto base station, create small wireless coverage area to connect user equipment to the cellular core network through the subscriber broadband internet access. Owing to the fact that, small cells are installed by the end-users, they are cost effective solutions as compared to other indoor coverage solutions. Since the femtocell users do not need to communicate with the macro base station, the battery life of the mobile devices is increased by femtocells [1]. Aiming at reducing cost and improving capacity, the co-channel deployment is preferred, so that the same portion of spectrum is used by both macro and femtocell base stations, and it causes an interference [3]. Therefore, the femto base stations provide better indoor coverage in the expense of causing interference [4]. Interference causes a strong degradation of both femtocell and macrocell user's signal-to-interference-plus-noise ratio (SINR).

The interference management has a great importance for femtocell networks to ensure that the users have good quality of service. Adding a new femtocell in the macro cell coverage area can disturb other users' functionality. Considering two-tier networks, interference can cause "dead zones" in other existing layer of network [5]. Dead zones can occur in both femtocell and macrocell coverage area due to the strong interference.

There are great deal of interference management techniques for femtocell networks. First, spectrum splitting has been suggested in [6]. In this technique, spectrum band is divided into two portions. One portion is allocated for macrocell while the other one is allocated for femtocell. However, this is not an efficient technique due to scarcity of spectrum. In some cases where dense deployment of femtocell is concerned, separate portion of spectrum can be used for femtocell operations [7]. Power control is also a key technique in the interference avoidance. In this technique, by controlling the transmit power of femtocell, macro user in the vicinity of the femtocell can be protected. It has been carried out by using game theory in [8], where macro and femto base stations operate in semi-autonomous mode in order to maximize energy-efficiency. In this scheme, each base station updates its power allocation strategy to maximize its utility. Another power control technique is based on Stackelberg game $[9,10]$. In the techniques, the macro base station acts a leader while femtocells act as a followers. The leader adjusts its power and imposes interference price on followers to maintain its own user's minimum data rate requirements. Subsequently, the followers optimize their powers based on the imposed price. The similar technique has been also presented based on game theoretical approaches in [11]. In order to achieve much more effective interference management technique, cognitive capabilities of femtocells can be used for interference mitigation [12]. In [13], an interference alignment for femtocell overlaid cellular network has been presented by selecting the strongest interfering nodes and applying interference cancellation at each node. In [14], a power allocation strategy has been examined for the cross-tier interference management problem in macrocell uplink communication and femtocell users.

In this paper, we propose a joint beamforming and power allocation technique for femtocell networks consists of one macro base station and multiple femto base stations 
with multiple transmit antennas. Our goal is to mitigate cross-tier interference and to design power efficient femtocell networks. Firstly, we apply interference cancellation by employing partial zero forcing to mitigate the strongest interference between different layers. Then, we perform power allocation to reduce power consumption and further mitigate interference while satisfying both macro and femto users' SINR requirements. The system model is introduced in Sect. 2. The proposed algorithm is described in Sect. 3. The performance evaluations and the conclusion are given in Sects. 4 and 5, respectively.

\section{System Model}

We consider a wireless network which consists of one macro base station with $N^{t m}$ transmit antennas and $K$ femto-base stations with $N^{t f}$ transmit antennas. Both the macro user and the femto user have one receive antenna.

In the femtocell network, our main target is to minimize the total transmit power of the femtocells while satisfying data rate requirements of both macro and femto users. The optimization problem is defined as in the following:

$$
\min \sum_{k} P_{k}^{f}
$$

subject to

$$
\begin{gathered}
\gamma^{m} \geq \gamma^{\text {mtar }} \\
\gamma_{k}^{f} \geq \gamma_{k}^{\text {ftar }} ; \quad \forall k \\
P^{\text {fmin }} \leq P_{k}^{f} \leq P^{\text {fmax }} ; \quad \forall k
\end{gathered}
$$

where $P_{k}^{f}$ is transmit power of the $k$ th femto base station, $\gamma^{\text {mtar }}$ and $\gamma_{k}^{\text {ftar }}$ are the target SINR for the macro user and the kth femto user, respectively. $P^{f m i n}$ and $P^{f m a x}$ are the minimum and maximum allowable transmit power of the femto base station.

The instantaneous SINR values of the macro user and the $k t h$ femto user are given as follows:

$$
\begin{gathered}
\gamma^{m}=\frac{\left(P^{t m} / P L^{m m}\right) G^{m m}}{\sum_{k=1}^{K}\left(P_{k}^{f} / P L_{k}^{f m}\right) G_{k}^{f m}+N_{0} B} \\
\gamma_{k}^{f}=\frac{\left(P_{k}^{f} / P L_{k, k}^{f f}\right) G_{k, k}^{f f}}{\sum_{j=1 ; j \neq k}^{K}\left(P_{j}^{f} / P L_{j, k}^{f f}\right) G_{j, k}^{f f}+\left(P^{t m} / P L_{k}^{m f}\right) G_{k}^{m f}+N_{0} B}
\end{gathered}
$$

where $P^{t m}$ is transmit power of the macro base station, $P L^{m m}, P L_{k}^{f m}, P L_{k, k}^{f f}$ and $P L_{k}^{m f}$ include the effect of path loss and shadowing between the macro user and the macro base station, between the macro user and the $k$ th femto base station, between the $k$ th femto user and the $k t h$ femto base station, between the $k t h$ femto user and the macro base station respectively, $N_{0}$ is power spectral density and $B$ is the total bandwidth. $G^{m m}$ is gain between the macro base station and the macro user, $G_{k}^{f m}$ is gain between the $k t h$ femto base station and the macro user, $G_{k, k}^{f f}$ is the one between the $k t h$ femto base station and the $k$ th femto user, $G_{j, k}^{f f}$ is 
the one between the $j t h$ femto base station and the $k t h$ femto user, $G_{k}^{m f}$ is the one between the macro base station and the $k t h$ femto user. We will determine these gain values by using either partial zero forcing or maximum ratio combining strategies in the following section.

The femtocells are located far from each other in the macro coverage. Therefore, we assume that the term for co-tier interference, $\sum_{j=1 ; j \neq k}^{K}\left(P_{j}^{f} / P L_{j, k}^{f f}\right) G_{j, k}^{f f}$ becomes zero and we only focus on cross-tier interference in this paper.

\section{The Proposed Joint Beamforming and Power Allocation Technique}

We perform a joint beamforming and power allocation technique into two phase. In this first phase, all femto base stations tune their maximum transmit power. The femto base station that causes the highest interference for the macro user is determined and then the partial zero forcing (PZF) beamforming [15] is applied to eliminate cross-tier interference. We perform maximum ratio combining (MRC) beamforming for all remaining femto base stations. At the macro base station, the PZF beamforming is applied to eliminate the interference on the femto user which receives the highest interference from the macro base station. At the second stage, we apply power allocation to design a power efficient femtocell networks and to further reduce the interference caused by the femto base stations. While performing power allocation, the total received interference power on the macro user from the femto base stations is calculated and the transmit power of the femto base stations is reduced proportional to the distances between the femto base stations and the macro user.

The femto user which receives the average highest interference from the macro base station is determined by,

$$
k^{\prime}=\arg \max _{k} P_{k}^{I_{f}}
$$

where $P_{k}^{I_{f}}=P^{t m} / P L_{k}^{m f}$.

Then, the beamforming vector for the macro base station is designed as,

$$
\mathbf{w}^{\prime m}=\left(\mathbf{I}-\mathbf{Z}^{m}\right) \mathbf{h}^{m m}
$$

where $\mathbf{h}^{m m}$ is the channel coefficient between the macro base station and the macro user, $\mathbf{Z}^{m}$ is the projection matrices on $\mathbf{V}^{m}$ and is formulated as

$$
\mathbf{Z}^{m}=\mathbf{V}^{m}\left(\left(\mathbf{V}^{m}\right)^{H} \mathbf{V}^{m}\right)^{-1}\left(\mathbf{V}^{m}\right)^{H}
$$

with

$$
\mathbf{V}^{m}=\mathbf{h}_{k^{\prime}}^{m f}
$$

where $\mathbf{h}_{k^{\prime}}^{m f}$ is the channel coefficient between the macro base station and the $k^{\prime}$ th femto user. Then, the beamforming vector for the macro base station is obtained by normalizing $\mathbf{w}^{m}=\frac{\mathbf{w}^{\prime m}}{\left\|\mathbf{w}^{\prime m}\right\|}$.

The femto base station which causes the highest interference to the macro user is determined by, 


$$
k^{*}=\arg \max _{k} P_{k}^{I}
$$

where $P_{k}^{I}=P^{t f} / P L_{k}^{f m}$.

Then, beamforming vector of the $k^{*}$ th femto base station is designed as,

$$
\mathbf{w}_{k^{*}}^{\prime f}=\left(\mathbf{I}-\mathbf{Z}_{k^{*}}^{f}\right) \mathbf{h}_{k^{*}, k^{*}}^{f f}
$$

where $\mathbf{Z}_{k^{*}}^{f}$ is the projection matrice on $\mathbf{V}_{k^{*}}^{f}$ and formulated as

$$
\mathbf{Z}_{k^{*}}^{f}=\mathbf{V}_{k^{*}}^{f}\left(\left(\mathbf{V}_{k^{*}}^{f}\right)^{H} \mathbf{V}_{k^{*}}^{f}\right)^{-1}\left(\mathbf{V}_{k^{*}}^{f}\right)^{H}
$$

with

$$
\mathbf{V}_{k^{*}}^{f}=\mathbf{h}_{k^{*}}^{f m}
$$

where $\mathbf{h}_{k^{*}}^{f m}$ is the channel coefficient between the macro user and the $k^{*}$ th femto user. Then, the beamforming vector of the $k^{*}$ th femto base station is obtained by $\mathbf{w}_{k^{*}}^{f}=\frac{\mathbf{w}_{k^{*}}^{\prime f}}{\left\|\mathbf{w}_{k^{*}}^{\prime f}\right\|}$.

For all remaining femto base stations, we apply MRC beamforming as,

$$
\mathbf{w}_{k}^{f}=\frac{\mathbf{h}_{k}^{f f}}{\left\|\mathbf{h}_{k}^{f f}\right\|} \quad \forall k ; k \neq k^{*}
$$

Then, the gains belonging to the macro base station and for all femto base stations are calculated by,

$$
\begin{gathered}
G^{m m}=\left|\left(\mathbf{h}^{m m}\right)^{H} \mathbf{w}^{m}\right|^{2} \\
G_{k}^{m f}=\left|\left(\mathbf{h}_{k}^{m f}\right)^{H} \mathbf{w}^{m}\right|^{2} ; \quad \forall k \\
G_{k, k}^{f f}=\left|\left(\mathbf{h}_{k}^{f f}\right)^{H} \mathbf{w}_{k}^{f}\right|^{2} ; \quad \forall k \\
G_{k}^{f m}=\left|\left(\mathbf{h}_{k}^{f m}\right)^{H} \mathbf{w}_{k}^{f}\right|^{2} ; \quad \forall k
\end{gathered}
$$

With the help of designed beamforming vectors, the gains $G_{k^{\prime}}^{m f}$ and $G_{k^{*}}^{f m}$ are forced to zero by producing beamforming vectors in a way that they are orthogonal to the cross-tier channel vectors while $G^{m m}$ and $G_{k, k}^{f f}$ are maximized. In this way, beamforming vectors enable the base stations to adjust their gain depending on users basis, which makes it possible to eliminate highest interference for both the femto user and the macro user while maximizing the channel gains.

After performing PZF and MRC beamforming, we propose to adjust transmit power of the femto base stations. The minimum femto base station power for the femto users can be determined based on the maximum and minimum transmit power bound while elimination cross-tier interference and satisfying both macro and SINR requirements of the femtousers. 
As a result of the first stage, the total received interference power for the macro user, $P_{I}^{m}$, is calculated to determine in which way each individual femto base station adjusts its power so that the femto base stations do not pose an interference to the macro user.

This aggregated average interference power is given by,

$$
P_{I}^{m}=\sum_{\forall k ; k \neq k^{*}}\left(P_{k}^{f} / P L_{k}^{f m}\right)
$$

Using Eq. (5), the maximum interference power bound is written as follows:

$$
P_{I}^{m}=\left[\frac{P^{t m}}{P L^{m m} \gamma^{m t a r}}-N_{0} B\right]
$$

The transmitted power is adjusted among the femto base stations in a distance-based manner.

$$
C_{k}^{f}=\frac{d_{k}}{\sum_{k=1 ; \forall k \neq k^{*}}^{K} d_{k}}
$$

where $d_{k}$ is the distance between the macro user and the kth femto base station.

This coefficient, $P_{I}^{m}$ and the path loss coefficient between femto base stations and macro user are used in adjusting the power of femtocells by,

$$
P_{k}^{f m a x}=C_{k}^{f} P_{I}^{m} P L_{k}^{f m} \quad \forall k ; k \neq k^{*}
$$

Thus, the transmit power for the femto base stations that causes interference to the macro user is adjusted proportional to the distance between the femto base station and the macro user. The power allocation by Eq. (23) provides a maximum power bound, which is denoted by $P_{k}^{f m a x}$, for the femtocells so that they do not cause interference on the macro user. For the $k^{*}$ th femto base station that is performed PZF to eliminate interference for the macro user, the allowed transmit power is equal to maximum transmit power for femto base station, $P^{f m a x}$.

Using Eq. (6), the minimum transmit power for the kth femto base station can be formulated by,

$$
P_{k}^{f \min }=\left[\frac{P^{t m}}{P L_{k}^{m f}}+N_{0} B\right] \gamma^{f t a r} P L_{k, k}^{f f} \quad \forall k ; k \neq k^{\prime}
$$

Since the cross-tier interference is the only considered, the co-tier interference term in SINR of the femto user is not included in Eq. (24).

For the $k^{\prime}$ th femto base station, since base station is performed PZF to eliminate interference for the $k^{\prime}$ th femto user, the transmit power is adjusted to satisfy its user' SINR constraint as follows:

$$
P_{k^{\prime}}^{f \min }=P L_{k^{\prime}, k^{\prime}}^{f f} \gamma^{f t a r} N_{0} B
$$

Equations (24), (25) provides that all femto users can satisfy their SINR' requirements. $P_{k}^{f m i n}$ is calculated to satisfy the users' SINR requirements in the network, $P^{f m a x}$ is determined considering the interference constraints.

These two parameters on maximum and minimum power level for femto base stations form a feasible power region by, 


$$
\max \left[P^{f \min }, P_{k}^{f m i n}\right] \leq P_{k}^{f} \leq \min \left[P^{f m a x}, P_{k}^{f m a x}\right] \quad \forall k ; k \neq k^{*}
$$

The proposed power allocation achieves not only to have good satisfaction ratio for all macro and femto users but also to design power efficient femtocell networks.

\section{Performance Evaluations}

In this section, the performance results are illustrated for the scenarios which include one macro base station and a single femto base station, four femto base stations and randomly placed multiple base stations in macro coverage area based on the simulation parameters listed in Table 1.

\subsection{Single Femto Base Station Case}

The joint beamforming and power control technique is of great use to the systems which are aiming at both interference-free environment and power-efficient networks. In order to illustrate the effects of this technique, firstly the performance results are obtained for the network including only one femto base station, given in Fig. 1.

In Fig. 2, the elimination of interference is shown depending on the distance between the femto base station at the coordinates of $(600,800)$ and the macro user. The SINR of the macro user is stabilized even though the macro user is very close to the femto base station and is not degraded with the help of the proposed joint technique.

The achievable power-efficiency is illustrated in Fig. 3 that gives comparison results for the only power control and the joint proposed technique based on power consumption. The femto base station and the macro user are at the coordinates of $(600,800)$ and $(800,620)$ respectively. The transmit power of the femto base station changes with respect to the distance of the femto user to the femto base station due to the controllable-nature of it. The

Table 1 Simulation parameters

\begin{tabular}{lll}
\hline Explanation & Parameters & Value \\
\hline Transmit power of the macro base station & $P^{t m}$ & $43 \mathrm{dBm}$ \\
Transmit power of the femto base station & $P^{f}$ & Variable \\
Maximum allowable transmit power for the femto base & $P^{f m a x}$ & $23 \mathrm{dBm}$ \\
$\quad$ station & & $0 \mathrm{dBm}$ \\
Minimum allowable transmit power for the femto base station & $P^{f m i n}$ & $10 \mathrm{~dB}$ \\
Target SINR values of the macro user & $\gamma^{\text {mtar }}$ & $10 \mathrm{~dB}$ \\
Target SINR values of the femto user & $\gamma^{f t a r}$ & $-174 \mathrm{dBm} / \mathrm{Hz}$ \\
Noise power spectral density & $N_{0}$ & $10 \mathrm{MHz}$ \\
Bandwidth & $B$ & $128.1+37.6 \log _{10}\left(d_{m}\left(\mathrm{~km}^{\mathrm{fm}}\right)\right)$ \\
Path Loss Model for the macro user & $P L^{m}$ & $37+18.3 n+30 \log _{10}\left(d_{f}(m)\right)$ \\
Path Loss Model for the femto user & $P L^{f}$ & $4 \mathrm{~dB}$ \\
Shadowing for the macro user & $\sigma_{m}^{2}$ & $2 \mathrm{~dB}$ \\
Shadowing for femto users & $\sigma_{f}^{2}$ & $1 \mathrm{~km}$ \\
Macro cell radius & $R^{m}$ & $100 \mathrm{~m}$ \\
Femto cell radius & $R^{f}$ &
\end{tabular}




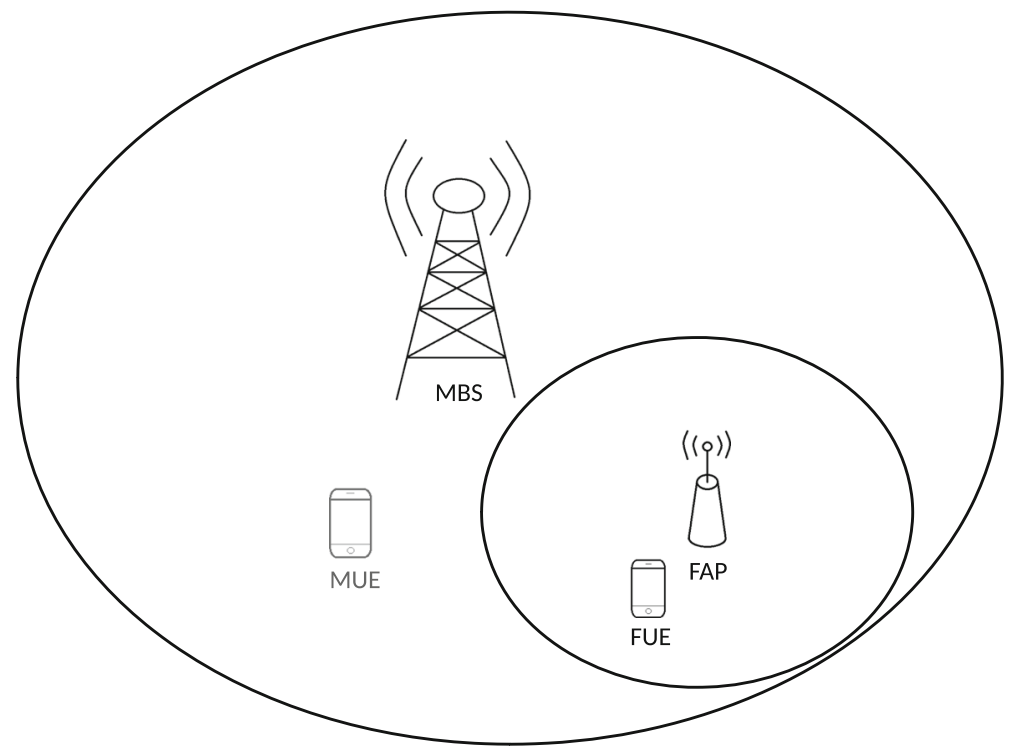

Fig. 1 The system model including a single femto-base station
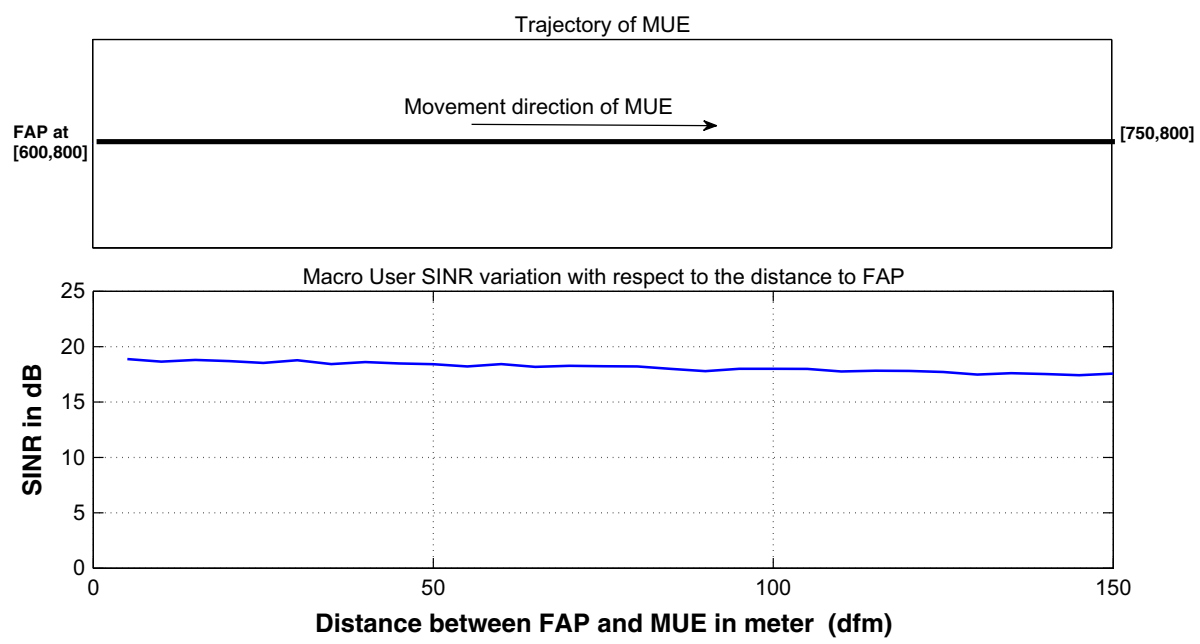

Fig. 2 SINR of the macro user with the proposed technique versus distance between the femto base station and the macro user for a single femto base station case

transmit power of the femto base station, when the joint technique is used, can be reduced around $15 \mathrm{~dB}$ compared to the only power control technique.

\subsection{Multiple Femto Base Stations Case}

In multiple femto base stations case, there are four femto base stations in macro coverage area as shown in Fig. 4. The macrocell base station is located in the origin and the femtocells are located in coordinate axis in a way that they constitute a square. 


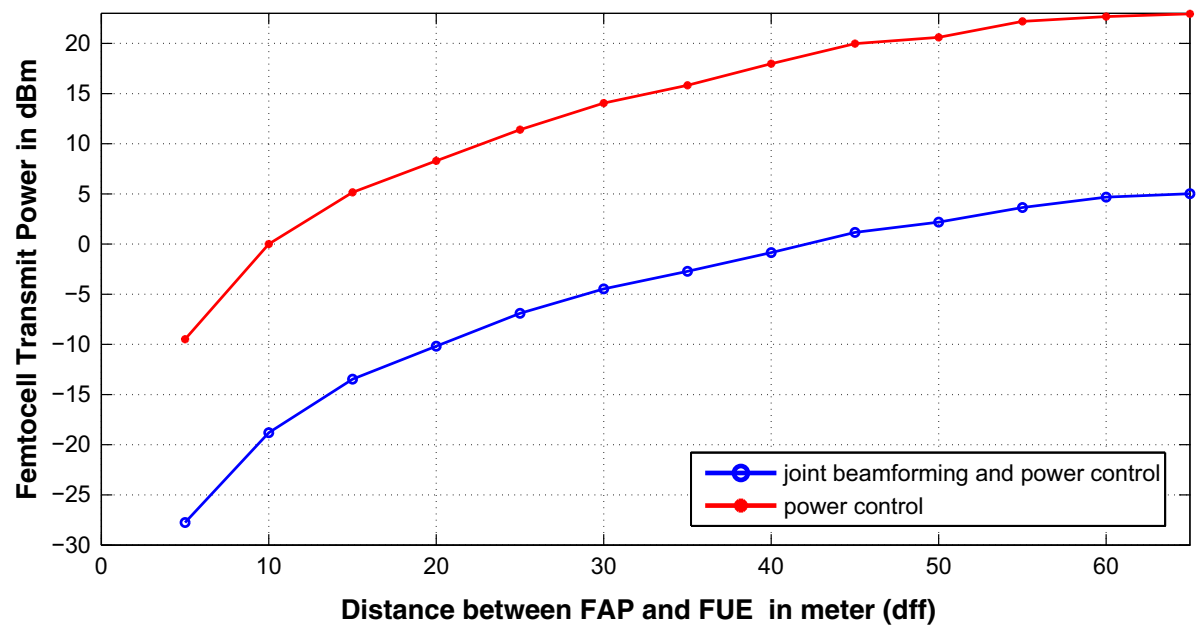

Fig. 3 Power consumption results of different algorithms for a single femto base station case

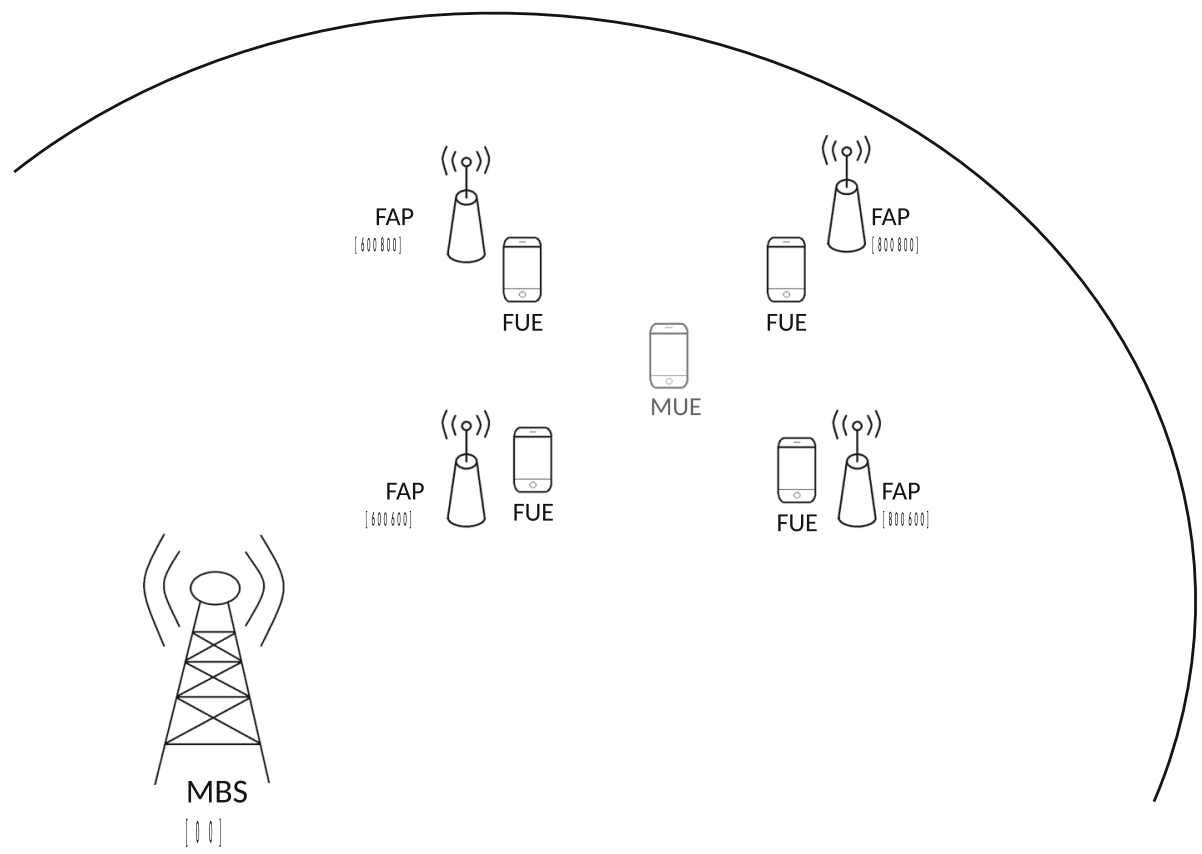

Fig. 4 The system model including multiple femto base stations

The beamforming technique is applied to the chosen femto base station which causes the greatest interference on the macro user and then power control technique is applied to all femto base stations as described in the proposed technique. At the macro base station, the beamforming technique is performed for the femto user that receives the highest interference from macro base station. We achieve both interference suppression and power efficiency with help of the proposed technique. 

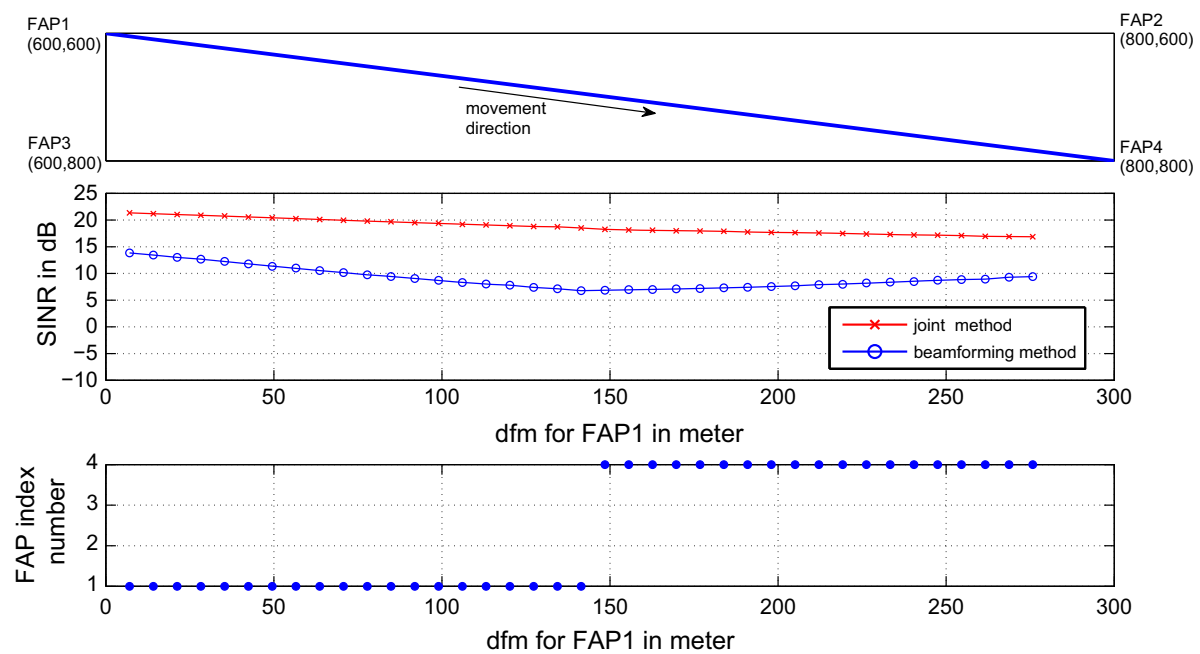

Fig. 5 SINR of the macro user moving from the femto base station 1 to 4 for the joint method

In Fig. 5, the SINR of the macro user with respect to its location is demonstrated for the proposed joint technique and the only beamforming technique. The first sub-figure, on the top of Fig. 5, illustrates the femto base stations layout in which the macro user moves toward to the determined-direction. The second sub-figure, in the middle of Fig. 5, shows the SINR changing and gives a comparable sight of SINR values for the two techniques. The third subfigure of Fig. 5 indicates which the femto base station is chosen at a given particular location of the macro user to implement the PZF beamforming. When the macro user is being close to the femto base station, its SINR does not undergoes any degradation effects, which proves that the joint technique suppresses the cross-tier interference successfully. Furthermore, a small amount of decreasing in SINRs of the macro user results from the fact that the macro user is moving away from the macro base station as seen in its trajectory graph.

In Fig. 6, the macro user moves over the different path compares to Fig. 5. In Fig. 6, the macro user is moving from 3rd femto base station, whose coordinate is $(600,800)$ to 2 nd femto base station along the diagonal whereas the macro user, in Fig. 5, is moving from 1st femto base station to 4rd femto base station. In both figures, x-axes of graphs represent, $\mathrm{dfm}$, the distance from the femto base station to the macro user. In Fig. 5, dfm denotes the distance from 1 st femto base station to the macro user while denoting the distance from $3 \mathrm{rd}$ femto base station to the macro user in Fig. 6.

The power distributions of the femto base stations in macro coverage area are given in Figs. 7 and 8. In these figures, the power of the femto base stations which are selected for beamforming process are not shown because they have no effects on the macro user. In Fig. 7, the macro user is located at the coordinates of $(690,610)$. Here, the 1 st femto base station is selected for beamforming method because it has a strongest interference power in this case. The 2nd femto base station has a lowest power because of its distance to the macro user. Since the distances of the other femto base stations are approximately the same, there is no observable difference between their powers. Moreover, Fig. 8 is the counterpart of the previous figure. The macro user is located at $(710,790)$. Here, 4rd femto base station is selected for beamforming process. Considering the distances of each femto base station to the macro user, the power sharing or controlling is successfully achieved for femto base stations as seen in Fig. 8. 

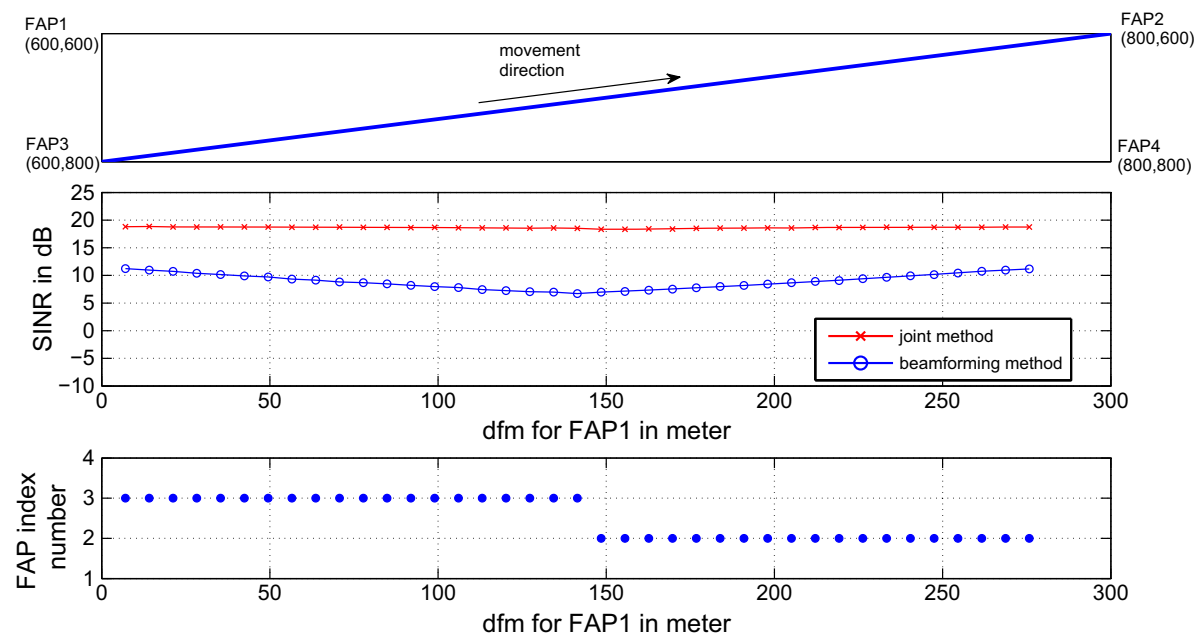

Fig. 6 SINR of the macro user moving from the femto base station 3 to 2 for the joint method

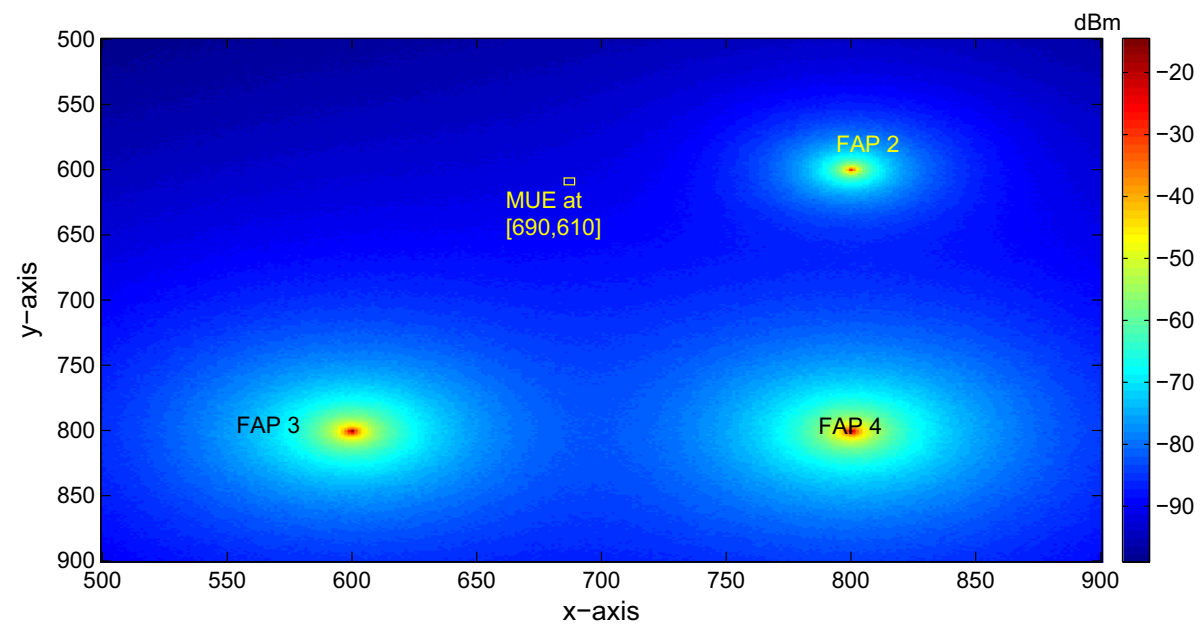

Fig. 7 Power distribution of the femto base stations for the macro user at $(690,610)$

In order to illustrate the gain of the proposed algorithm based on power consumption, we consider different number of femto base stations that are randomly located in the macro cell coverage area by taking into account the distances between them to hold the assumption of very low interference between co-tier network. The macro cell user and the femtocell users are randomly placed in the macro cell and their corresponding femtocell coverage, respectively. The macro base station is also adjusted its power depending on the location of the macro user to satisfy its SINR requirement. We illustrate the performance results of the joint proposed algorithm in terms of total average transmitted power by femto base stations compared to only the power control scheme in Fig. 9. It is clearly seen that total consumed power, when joint technique is employed, about $3 \mathrm{~dB}$ less than that of the only power control method for the multiple femto base stations case. 


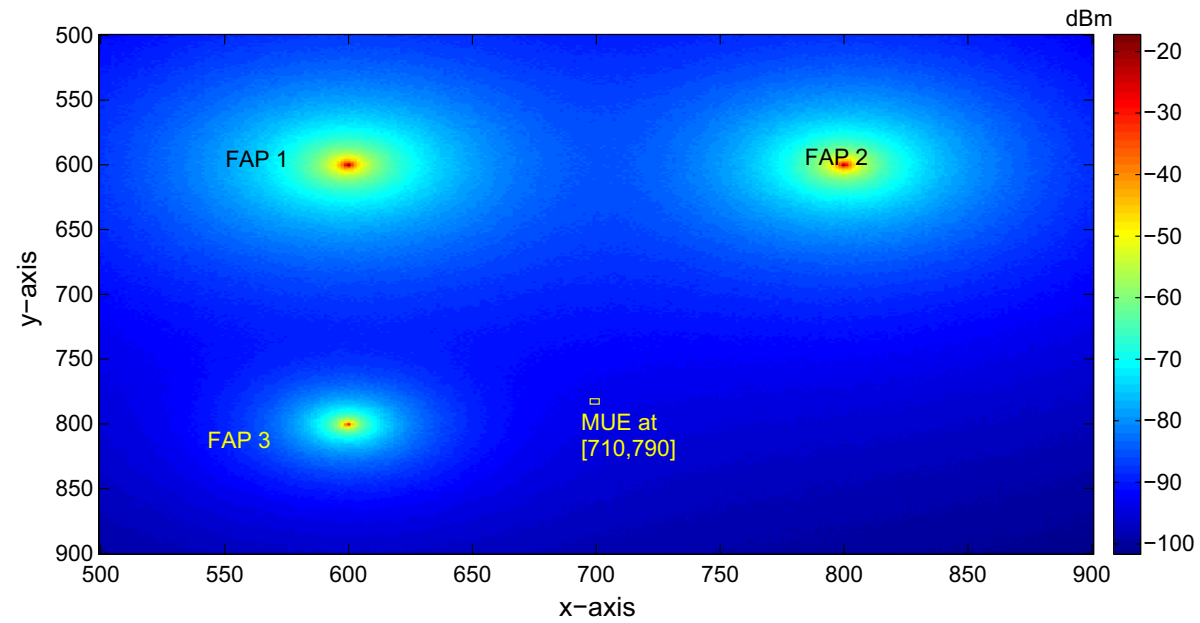

Fig. 8 Power distribution of the femto base stations for the macro user at $(710,790)$

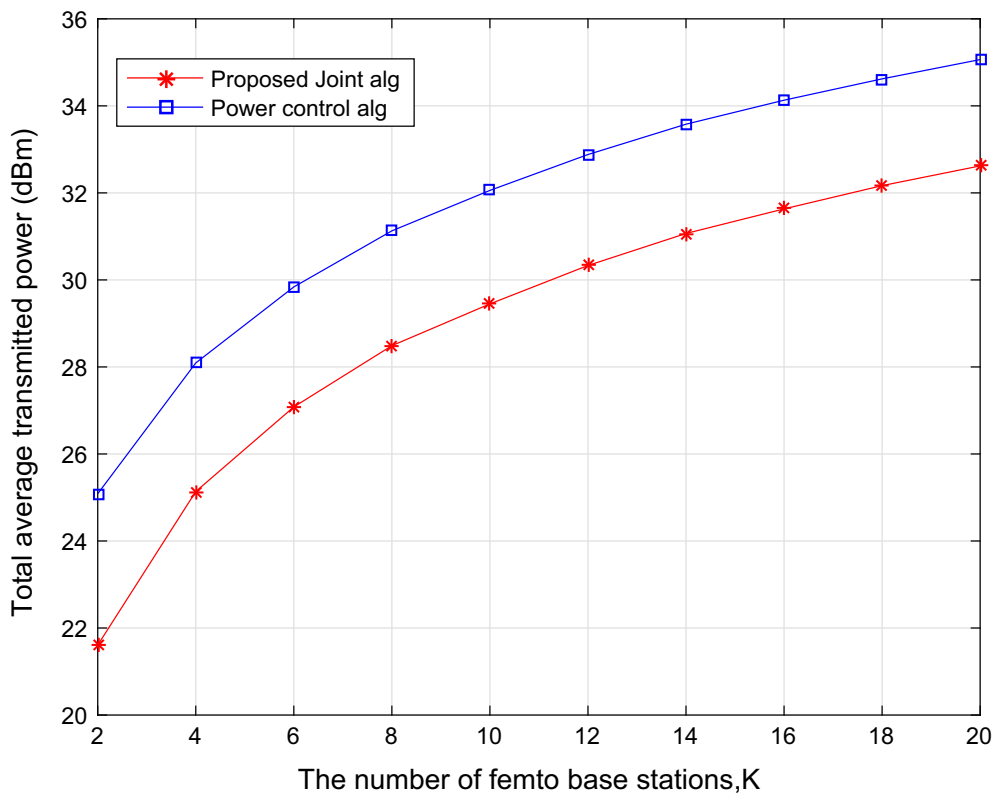

Fig. 9 Average total power consumption by femtocell network for different number of femto base stations

\section{Conclusion}

In this paper, interference management techniques including beamforming and power control have been proposed for two-tier networks consists of multiple femto base stations and a single macro base station.

In single femto base station case, the PZF beamforming cancel the interference signal while the power control enables femto base station to select minimum transmit power. As a 
result of this, it has been shown that these two techniques, when applied jointly, not only provide cross-tier interference suppression but also enable femtocell to consume less power than before. Thus, the proposed joint technique proves to be a power-efficient interference management solution when compared to individual techniques. In multiple femto base station case, the proposed joint technique has been applied differently. The PZF beamforming method has been applied to one femto base station which causes the strongest interference on the macro user, and the power allocation has been performed for all remaining femto base stations to further cancel interference and reduce the power consumption. The proposed technique works efficiently in terms of avoiding interference on the macro user and adjusting the power of the multiple femto base stations which are located randomly in the macro cell coverage area. According to the performance results on power consumption, the proposed joint beamforming and power control algorithm outperforms to the only power control algorithm significantly for the practical femtocell deployment scenarios.

As future femtocell networks, there is no doubt that interference management will eventually become the major concern because of, likely, dense deployment of the femto base stations. We will extend our proposed scheme for the dense deployment scenario, which also requires interference management between femtocells. Furthermore, we will consider the imperfect channel state information for the proposed joint power control and beamforming technique by designing an efficient limited feedback link for the femtocell networks.

\section{References}

1. Saquib, N., Hossain, E., Le Bao, L., \& Kim, D. I. (2012). Interference management in OFDMA femtocell networks: Issues and approaches. IEEE Wireless Communications, 19, 86-95.

2. Yavuz, M., Meshkati, F., Nanda, S., Pokhariyal, A., Johnson, N., Roghothaman, B., et al. (2009). Interference management and performance analysis of umts/hspa+ femtocells. IEEE Communications Magazine, 47(9), 102-109.

3. Chandrasekhar, V., \& Andrews, J. (2008). Femtocell networks: A survey. IEEE Communication Magazine, 46(9), 59-67.

4. Zahir, T., Arshad, K., Nakata, A., \& Moessner, K. (2013). Interference management in femtocell. IEEE Communications Surveys and Tutorials, 15, 293-311.

5. Lopez-Perez, D., Valcarce, A., de la Roche, G., \& Zhang, J. (2009). OFDMA femtocells: A roadmap on interference avoidance. IEEE Communication Magazine, 47, 41-48.

6. Yang, L., Song, S.H., \& Letaief, K.B. (June 2014). Cognitive spectrum access in two-tier femtocell networks. In IEEE International Communications Conference, Sydney, pp. 5354-5359, 10-14.

7. Chen, D., Jiang, T., \& Zhang, Z. (2014). Frequency partitioning methods to mitigate cross-tier interference in two-tier femtocell networks. IEEE Transactions on Vehicular Technology, 64, 1793-1805.

8. Mao, T., Feng, G., Liang, L., Qin, S., \& Wu, B. (2015). Distributed energy-efficient power control for macro-femto networks. IEEE Transactions on Vehicular Technology, 65, 718-731.

9. Ahmad, I., Feng, Z., Hameed, A., Zhang, P., \& Zhao, Y. (2014). Spectrum sharing and energy-efficient power optimization for two-tier femtocell networks. In 9th International conference on cognitive radio oriented wireless networks and communications (CROWNCOM), Oulu, pp. 156-161, 2-4.

10. He, S., Zhang, L., Wen, X., Zhang, Z., Lu, Z., \& Sun, Y. (2014). Price-based power control with statistical delay QoS guarantee in two-tier femtocell networks. In 21st International conference on telecommunications (ICT), Lisbon, pp. 318-322, 4-7.

11. Tseng, C. C., Peng, C. S., Lo, S. H., Wang, H. C., Kuo, F. C., \& Ting, K. C. (2014). Co-tier uplink power control in femtocell networks by Stackelberg game with pricing. In 4th International conference on wireless communications, vehicular technology, information theory and aerospace and electronic systems (VITAE), Aalborg, pp. 1-5, 11-14.

12. Kpojime, H. O., \& Safdar, G. A. (2014). Interference mitigation in cognitive radio based femtocells. IEEE Communications Surveys and Tutorials, 17, 1511-1534. 
13. Ghiamatyoun, A., \& Olfat, A. (2014). Downlink interference alignment in two-tier femtocell networks. Wireless Personal Communications, 82, 777-798.

14. Han, Q., Ma, K., Liu, Z., \& Guan, X. (2013). Power control based on maximum power adaptation in two-tier femtocell networks. Wireless Personal Communications, 70, 331-335.

15. Jindal, Q. N., Andrews, J. G., \& Weber, S. (2009). Rethinking MIMO for wireless networks: Linear throughput increases with multiple receive antennas. In Proceedings of IEEE International Conference on Communications (ICC), Dresden, Germany.

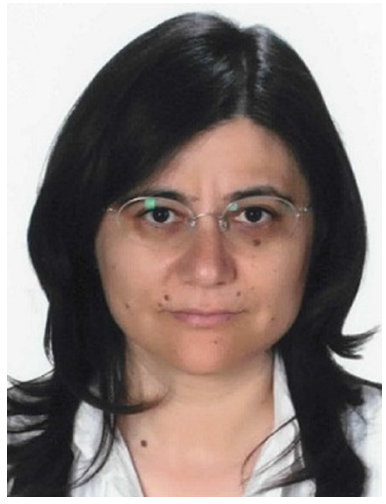

Berna Özbek is graduated from Electrical and Electronics Department of Dokuz Eylul University, Turkey on 1994 and completed her MSc and Ph.D. studies respectively on 1999 and 2004. Afterwards, she received a scholarship and worked as a postdoctoral researcher at CNAM-Paris on 2005. Currently, she holds an Assistant Professor position in Telecommunication field at the Electrical and Electronics Engineering Department of İzmir Institute of Technology, Turkey and is working in the field of wireless communication systems for more than ten years. She was awarded as a Marie-Curie Intra-European (EIF) Fellow by European Commission on 2010 and worked in the project entitled Interference Management Techniques for Multicell Networks for two years in CNAM-Paris. Her research interests are on interference management strategies, limited feedback strategies, D2D based communications, multiuser multi-antenna multicarrier systems. She has managed 1 international and 4 national projets and served as a consultant for 3 Eureka-Celtic projects. She is serving as a referee for many international journals and conferences, European Commission project, Turkish Republic of Ministry of Trade and Industry and The Scientific and Technological Research Council of Turkey. Under her supervision, 8 master and 2 doctoral dissertations have been completed. She has an author of more than 60 peer-reviewed papers, 1 book chapter, 1 book and 2 patents.

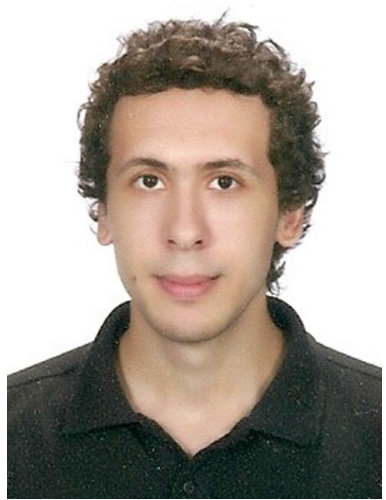

Ŭür Bayrak is gratuated from Electrical and Electronics Department of Ege University, Turkey in 2011 and completed his MSc study in 2015 from İzmir Institute of Technology. Afterward, he started a job in ITS telecommunication company as a Network Engineer in Izmir. Currently, he is continuing his career at Nokia Turkey office, Istanbul, in Fixed Access Network Technical Support Department. He is working on the realms of GPON and xDSL access networks. His research interests are on GPON and xDSL technologies, Fixed Access Network models, interference management strategies in Femtocells. 